\title{
A cross-sectional analysis of cardiovascular disease in the hemophilia population
}

\author{
Suman L. Sood, ${ }^{1}$ Dunlei Cheng, ${ }^{2}$ Margaret Ragni, ${ }^{3}$ Craig M. Kessler, ${ }^{4}$ Doris Quon, ${ }^{5}$ Amy D. Shapiro, ${ }^{6}$ Nigel S. Key, \\ Marilyn J. Manco-Johnson, ${ }^{8}$ Adam Cuker, ${ }^{9}$ Christine Kempton, ${ }^{10}$ Tzu-Fei Wang, ${ }^{11}$ M. Elaine Eyster, ${ }^{12}$ Philip Kuriakose, ${ }^{13}$ \\ Annette von Drygalski, ${ }^{14}$ Joan Cox Gill, ${ }^{15}$ Allison Wheeler, ${ }^{16}$ Peter Kouides, ${ }^{17}$ Miguel A. Escobar, ${ }^{18}$ Cindy Leissinger, ${ }^{19}$ \\ Sarah Galdzicka, ${ }^{20}$ Marshall Corson, ${ }^{21}$ Crystal Watson, ${ }^{2}$ and Barbara A. Konkle ${ }^{20,21}$ \\ ${ }^{1}$ Department of Internal Medicine, University of Michigan, Ann Arbor, MI; ${ }^{2}$ American Thrombosis and Hemostasis Network, Chicago, IL; ${ }^{3}$ Department of Medicine, University of \\ Pittsburgh, Pittsburgh, PA; ${ }^{4}$ Department of Medicine, Georgetown University, Washington, DC; ${ }^{5}$ Orthopedic Hospital, Los Angeles, CA; ${ }^{6}$ ndiana Hemophilia and Thrombosis \\ Center, Indianapolis, IN; ${ }^{7}$ Department of Medicine, University of North Carolina, Chapel Hill, NC; ${ }^{8}$ Department of Pediatrics, University of Colorado, Anschutz Medical \\ Campus, Aurora, CO; ${ }^{9}$ Department of Medicine, University of Pennsylvania, Philadelphia, PA; ${ }^{10}$ Departments of Medicine and Pediatrics, Emory University, Atlanta, GA; \\ ${ }^{11}$ Department of Medicine, The Ohio State University, Columbus, OH; ${ }^{12}$ Department of Medicine, Penn State Milton S. Hershey Medical Center, Hershey, PA; ${ }^{13}$ Department \\ of Medicine, Henry Ford Hospital, Detroit, MI; ${ }^{14}$ Department of Medicine, University of California, San Diego, CA; ${ }^{15}$ Blood Center of Wisconsin, Milwaukee, WI; \\ ${ }^{16}$ Department of Medicine, Vanderbilt University, Nashville, TN; ${ }^{17}$ Mary M. Gooley Hemophilia Center, Rochester, NY; ${ }^{18}$ Department of Medicine, University of Texas \\ Health Science Center and McGovern Medical School, Houston, TX; ${ }^{19}$ Department of Medicine, Tulane University, New Orleans, LA; ${ }^{20}$ Bloodworks Northwest, Seattle, WA; \\ and ${ }^{21}$ Department of Medicine, University of Washington, Seattle, WA
}

\section{Key Points}

- $\mathrm{CV}$ risk factors are common in older men with hemophilia.

- Although older men with hemophilia have less CV disease than comparable unaffected men, CV events do occur and require treatment.
Men with hemophilia were initially thought to be protected from cardiovascular disease (CVD), but it is now clear that atherothrombotic events occur. The primary objective of the CVD in Hemophilia study was to determine the prevalence of CVD and CVD risk factors in US older men with moderate and severe hemophilia and to compare findings with those reported in age-comparable men in the Atherosclerosis Risk in Communities (ARIC) cohort. We hypothesized if lower factor levels are protective from CVD, we would see a difference in CVD rates between more severely affected and unaffected men. Beginning in October 2012, 200 patients with moderate or severe hemophilia A or B (factor VIII or IX level $\leq 5 \%$ ), aged 54 to 73 years, were enrolled at 19 US hemophilia treatment centers. Data were collected from patient interview and medical records. A fasting blood sample and electrocardiogram (ECG) were obtained and assayed and read centrally. CVD was defined as any angina, any myocardial infarction by ECG or physician diagnosis, any self-reported nonhemorrhagic stroke or transient ischemic attack verified by physicians, or any history of coronary bypass graft surgery or coronary artery angioplasty. CVD risk factors were common in the population. Compared with men of similar age in the ARIC cohort, patients with hemophilia had significantly less CVD (15\% vs $25.8 \%$; $P<$.001). However, on an individual patient level, CVD events occur and efforts to prevent cardiovascular events are warranted. Few men were receiving secondary prophylaxis with low-dose aspirin, despite published opinion that it can be used safely in this patient population.

\section{Introduction}

Hemophilia $\mathrm{A}$ and $\mathrm{B}$ are $\mathrm{X}$-linked disorders that result in bleeding as the result of an absence or decrease in coagulation factor VIII or factor IX, respectively. Hemophilia is classified as severe $(<1 \%$ clotting factor activity), moderate (1\%-5\%), and mild (6\%-40\%). ${ }^{1}$ Without treatment, patients with hemophilia, particularly those with severe disease, have recurrent disabling and life-threatening bleeding episodes. More recent use of prophylactic factor therapy to prevent bleeding has changed the landscape of 
hemophilia, and patients with hemophilia are living longer because of improvements in treatment and general medical care. ${ }^{2-4}$

Among 14990 US men with hemophilia A or B reported in September 2017 in the American Thrombosis and Hemostasis Network data set, $14.5 \%$ were aged 50 years or older. ${ }^{5}$ As cardiovascular disease (CVD) is a disease that increases with age, these men are at risk for cardiovascular complications. Men with hemophilia were initially thought to be protected from CVD, but it is now clear that atherothrombotic events do occur. ${ }^{6-13}$ Limited data suggest that hemophilia is not protective against atherosclerosis, ${ }^{13-15}$ but this has not been well studied in men with severe hemophilia without other CVD risk factors, such as active HIV infection. CVD risk factors are prevalent in the hemophilia population, and for reasons not understood, hypertension appears to be more common, even in younger men. ${ }^{16,17}$ Overall, most, but not all, studies have shown some protection from CVD in the hemophilia population. ${ }^{8}$ In general, these studies have evaluated patients with all severities of hemophilia and have not focused on those with moderate and severe disease, who in theory may be most protected from CVD.

The primary objective of the CVD in Hemophilia study reported here was to determine the prevalence of CVD and CVD risk factors in older men with moderate and severe hemophilia in the United States. In addition, we compared the prevalence of CVD and CVD risk factors in our hemophilia cohort with age-comparable men in the US Atherosclerosis Risk in Communities (ARIC) and National Health and Nutrition Examination Survey (NHANES) cohorts. CVD was defined as any angina validated by the Rose questionnaire, ${ }^{18}$ any myocardial infarction (MI) by electrocardiogram (ECG) or physician diagnosis, any self-reported nonhemorrhagic stroke or transient ischemic attack that was verified by physicians, or any history of coronary bypass graft surgery or coronary artery angioplasty.

\section{Methods}

The CVD in Hemophilia study was a US national cross-sectional study, which began enrollment in October 2012. Patients were enrolled at 19 hemophilia treatment centers (HTCs) in the United States. The study included men with moderate or severe congenital hemophilia A or B (factor VIII [FVIII] or IX level $\leq 5 \%$ ) aged 54 to 73 years. The age range was established to allow data to be compared with that in the ARIC study. ${ }^{19}$ The enrollment goal of 200 subjects was determined on the basis of $80 \%$ power to show a difference in the prevalence of CVD compared with the same-aged ARIC cohort, assuming the prevalence of CVD in the subjects with hemophilia was $14.9 \%$ (vs $23 \%$ in ARIC). Men with an additional bleeding disorder (besides liver dysfunction) were excluded. The study was approved by the local Institutional Review Board at each site.

Men with mild hemophilia (FVIII or FIX level 6\%-40\%) were excluded because, with our hypothesis that a low factor activity level is protective from CVD, we would expect to see a greater difference in CVD between men with normal factor levels and those with more severely decreased factor levels. Patients with mild disease would be at intermediate risk, and a larger number of patients would be needed to compare CVD events between those with mild and more severe disease or between those with mild disease and nonhemophilic control patients.

After obtaining written informed consent, data were collected from patient interview and medical records and included CVD risk factors, medications, and history of thrombotic events. A fasting blood sample was collected and assayed centrally (University of Washington, Seattle, WA) for glucose, lipids, creatinine, and highsensitivity C-reactive protein (hsCRP). A 12-lead ECG was performed and read centrally and independently by 2 masked cardiologists. Evidence of an Ml was defined by the presence of Minnesota Code (MC) classifications as a major Q/QS abnormality (MC 1.1 or 1.2) or minor Q/OS abnormality (MC 1.3) and major ST-T abnormality (MC $4.1,4.2,5.1$, or 5.2).$^{20}$ If the independent ECG readings differed, a final reading was given by consensus. CVD was defined as any angina validated by the Rose questionnaire, ${ }^{18}$ any MI by ECG or physician diagnosis, any self-reported nonhemorrhagic stroke or transient ischemic attack that was verified by physicians, or any history of coronary bypass graft surgery or coronary artery angioplasty. Additional definitions used were prophylaxis, regular infusions of FVIII ( $\geq 2$ doses/ week) or FIX ( $\geq 1$ dose/week) to prevent bleeding (3 patients receiving prophylaxis were receiving extended half-life products); obesity, body mass index higher than $30 \mathrm{~kg} / \mathrm{m}^{2}$; elevated waist circumference, higher than $102 \mathrm{~cm}$; hypertension, sitting systolic blood pressure higher than $140 \mathrm{~mm} \mathrm{Hg}$, diastolic blood pressure higher than $90 \mathrm{~mm} \mathrm{Hg}$ (elevated levels were confirmed with repeat testing $\geq 30$ minutes later) or receiving antihypertensive medications; dyslipidemia, elevated fasting total cholesterol (>200 mg/dL), triglycerides (>150 mg/dL), lowdensity lipoprotein (LDL) (>130 mg/dL), decreased fasting HDL $(<40 \mathrm{mg} / \mathrm{dL})$, or receiving cholesterol lowering agents; diabetes mellitus, subject reported, elevated fasting glucose ( $>125 \mathrm{mg} / \mathrm{dL})$, or receiving medication; and elevated hsCRP (>1.0 mg/dL). Angina was defined using the validated Rose questionnaire as the presence of chest pain on exertion that was relieved within 10 minutes after stopping or slowing down. ${ }^{18} \mathrm{~A}$ positive family history of CVD was defined as a sibling or parent with a heart attack or stroke.

Male participants in the ARIC study were used as the primary comparison population. This CVD in Hemophilia study was structured using the same CVD definition as was used in ARIC, except that hemorrhagic stroke was not specifically excluded. The ARIC study is an National Institutes of Health-funded prospective epidemiologic study to investigate the etiology and natural history of atherosclerosis and cardiovascular disease in 4 US communities. ${ }^{19}$ The cohort component of the study was begun in 1987, and in the fourth and last follow-up period in 1996 to 1998,4826 men were aged 54 to 73 years, which is the age focus of this CVD in Hemophilia study. The ARIC cohort was chosen as a control because of the type and focus of its data collection and an age range comparable to that in which individuals frequently are diagnosed with CVD, and for which the hemophilia population size was sufficiently feasible to conduct this study. Peripheral vascular disease was not included in our definition of CVD because it was not part of the ARIC definition. Peripheral vascular disease includes pain with walking alleviated with rest, which can also occur in older men with hemophilia resulting from joint disease. We did not have resources for an objective measure of peripheral vascular disease, such as ankle brachial index.

To compare CVD prevalence in patients with hemophilia with a more contemporaneous population cohort, we used the NHANES 2013 to 2014 examination (https://wwwn.cdc.gov/nchs/nhanes). NHANES is a US population-based cross-sectional survey conducted by the National Center of Health Statistics at the Centers for Disease Control and Prevention. The study collects survey data on health and nutritional status of noninstitutionalized civilians of the US population through a series of interviews, examinations, and laboratory measurements. Participants are selected using a random sampling method, designed 


\begin{tabular}{|c|c|c|c|}
\hline & Hemophilia A, N (\%) & Hemophilia B, N (\%) & Total, N (\%) \\
\hline Severe disease $(F A<1 \%)^{*}$ & $79(64.2)$ & $32(41.6)$ & $111(55.5)$ \\
\hline Moderate disease (FA 1-5\%) & $44(35.8)$ & $45(58.4)$ & $89(44.5)$ \\
\hline Race: white/African American/other & $106(86.2) / 17(13.8) / 0$ & $71(92.2) / 4(5.2) / 2(2.6)$ & $177(88.5) / 21(10.5) / 2(1)$ \\
\hline Hispanic ethnicity & $5(4.1)$ & $5(6.5)$ & $10(5)$ \\
\hline Mean/median age, years & $61 / 60$ & $61 / 61$ & $61 / 60$ \\
\hline Use of prophylaxis & $42(34.2)$ & $19(24.7)$ & $61(30.5)$ \\
\hline Current inhibitor & $8(6.5)$ & $1(1.3)$ & $9(4.5)$ \\
\hline $\mathrm{HCV}^{*},+$ & $85(69.1)$ & $39(50.7)$ & $124(62)$ \\
\hline $\mathrm{HBV}^{*}, \neq$ & $6(4.9)$ & $2(2.6)$ & $8(4)$ \\
\hline $\mathrm{HIV}^{*}$ & $46(37.4)$ & $8(10.4)$ & $54(27)$ \\
\hline
\end{tabular}

FA, factor activity.

${ }^{*}$ Statistically significant differences between hemophilia A and B: severity, $P=.002 ; \mathrm{HCV}$ infection, $P=.009 ; \mathrm{HIV}$ infection, $P<.001$.

tIncludes those with active and treated infections.

flncludes those with HBV surface antigen and/or DNA positivity.

to oversample certain populations, including Hispanics, non-Hispanic African Americans, and non-Hispanic Asians. Of note, in NHANES, cardiac events are by history only, and ECGs are not obtained; transient ischemic attack (TIA) is also not recorded. A total of 862 men were chosen because their age at interview was 54 to 73 years, with a cohort mean age of 63 (standard deviation, 6) years. As per its design, NHANES had a higher percentage of Hispanic, African American, and Asian participants than our hemophilia cohort.

\section{Statistical analysis}

For categorical variables, $x^{2}$ or Fisher's exact tests were used to compare clinical and laboratory characteristics in those with hemophilia vs nonhemophilic men in the ARIC and NHANES studies. A logistic regression model using multivariate analysis followed by bivariate analyses was used to identify CVD risk factors in male patients with hemophilia, as well as to compare the prevalence of CVD between hemophilia and ARIC cohorts. The bivariate analyses built a foundation of covariates to be considered in the multiple logistic regression analysis. The models presented in the Results have the smallest Akaine Information Criterion or Bayesian Information Criterion values that best explain the data.

\section{Results}

Patients meeting all the inclusion criteria and none of the exclusion criteria were approached at each HTC. Two hundred one subjects were enrolled from 2012 to 2015 . One subject had a baseline factor activity level above $5 \%$ and was excluded from the analysis. Approximately $60 \%$ had hemophilia $A$, and just over half had severe disease; of the $40 \%$ with hemophilia B, $42 \%$ had severe disease. Patients with hemophilia A were more likely to have severe disease $(P=.002)$ and to have hepatitis $\mathrm{C}(P=.009)$ and HIV $(P<.001)$ infections than those with hemophilia $B$. African American subjects were more likely to have severe hemophilia than white subjects (76.2\% vs $52.5 \% ; P=.039)$. Almost one-third of patients were receiving routine prophylaxis with factor therapy. Patient demographics and baseline clinical characteristics are shown in Table 1. Approximately $60 \%$ of patients had been infected with hepatitis C and $27 \%$ with HIV. Forty-three (21.5\%) subjects had been diagnosed with cirrhosis, of whom $11(5.5 \%)$ had an elevated prothrombin time and $21(10.5 \%)$ had thrombocytopenia. Fifty-two of the 54 HIVpositive patients had a recent CD4 count available, with a median count of $484 / \mathrm{mm}^{3}$ (mean, $514 / \mathrm{mm}^{3}$; range, $86-867 / \mathrm{mm}^{3}$ ). Only 4 patients had CD4 counts below $200 / \mathrm{mm}^{3}$.

Cardiovascular risk factors were common in the population (Table 2). More than half the individuals had dyslipidemia and hypertension, and nearly one-third were obese. Although the mean creatinine was 1.1 $\mathrm{mg} / \mathrm{dL}$ (standard deviation, $0.4 \mathrm{mg} / \mathrm{dL}$ ), $26.8 \%$ of the subjects had an elevated value. Of the 119 subjects with hypertension, 72 (56\%) used antihypertensive medication. Of the 97 subjects who ever smoked, 32 were current smokers. African American patients were less likely to have dyslipidemia (38.1\% vs $66.1 \% ; P=.012)$, but other risk factors were similar between races.

In total, 30 subjects met criteria for CVD (defined as angina, MI, TIA, or ischemic or embolic stroke), for a prevalence of $15 \%$. Fifteen subjects (7.5\%) had MI ( 8 diagnosed by ECG only), $16(8 \%)$ had prior angina, 3 had a history of a TIA, and 1 had a history of an ischemic or embolic stroke. In addition, 15 subjects had atrial fibrillation/flutter, 6 had coronary stent placement, 3 had coronary bypass grafting, and 6 had venous thromboembolism ( 2 had both deep vein thrombosis and pulmonary embolism, 2 had deep vein thrombosis only, and 2 had pulmonary embolism only). As

\section{Table 2. CVD risk factors}

\begin{tabular}{lc}
\hline Risk factor & N (\%) \\
\hline Dyslipidemia & $133(65.8)$ \\
\hline Hypertension & $130(64.4)$ \\
\hline Does not engage in at least moderate physical activity* & $110(54.5)$ \\
\hline Ever smoked & $97(48.5)$ \\
\hline Family history of CVD & $87(43.6)$ \\
\hline Obesity (BMl $\left.\geq 30 \mathrm{~kg} / \mathrm{m}^{2}\right)$ & $59(29.5)$ \\
\hline Increased waist circumference $(>102 \mathrm{~cm})$ & $60(30)$ \\
\hline Diabetes mellitus & $39(19.5)$ \\
\hline Elevated hsCRP & $19(9.8)$ \\
\hline
\end{tabular}

BMI, body mass index.

*Moderate physical activity defined as $>90 \mathrm{~min} /$ week 


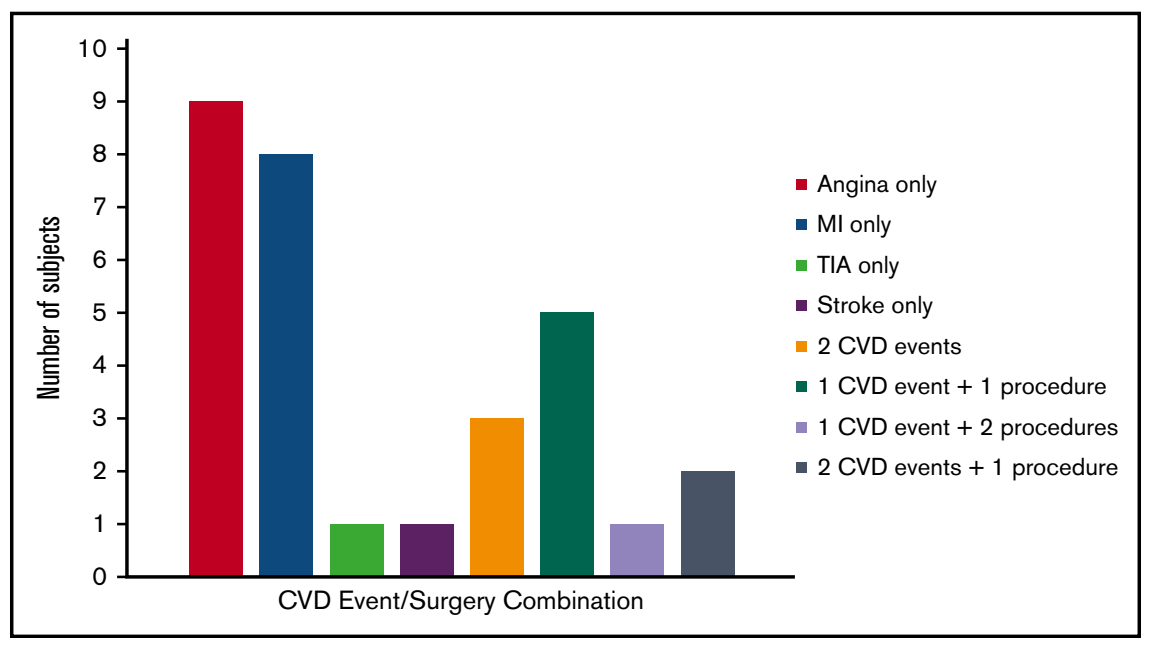

Figure 1. The distribution of CVD events and procedures among 30 subjects with CVD. The number of subjects who experienced CVD events (angina, MI, TIA, or stroke) alone or in combination with other events or procedures (coronary artery bypass grafting or angioplasty).

demonstrated in Figure 1, among the 30 subjects, 19 had a single CVD event, 3 had 2 CVD events, and 8 had at least 1 CVD event plus 1 CVD surgery.

On bivariate analysis, the development of CVD among the patients with hemophilia was significantly associated with hypertension $(P=$ $.002)$, dyslipidemia $(P=.014)$, and ever smoking $(P=.031)$. CVD risk factors for obesity, increased waist circumference, elevated hsCRP, and not engaging in physical activity were not associated with CVD in this population ( $P>.8$ for all). Hemophilia type (A vs $B$ ), severity (severe vs moderate), and treatment regimen (prophylaxis vs on-demand) had no significant associations $(P$ values $=.160$ and .291 , respectively) in this analysis. A multiple logistic regression model was built to describe the relationship among the hemophilia cohort between the presence of CVD and a priori chosen explanatory variables including CVD risk factors (age, race, family history of CVD, smoking, hypertension, diabetes, dyslipidemia) and hemophilia characteristics (type, severity, inhibitor presence, hepatitis $\mathrm{C}$ virus [HCV] and HIV infections, and interaction between severity and HCV). Among generally known CVD risk factors, nonwhite race, dyslipidemia, hypertension, and ever smoking all were significantly associated with CVD (Table 3). Among hemophilia characteristics, the presence of an inhibitor was significantly associated with CVD in the total cohort. In the absence of hepatitis $\mathrm{C}$, moderate hemophilia was significantly associated with CVD compared with severe hemophilia.

Compared with men of similar age in the ARIC cohort, patients with hemophilia had significantly less CVD (15\% vs $25.8 \%$; $P<.001)$. Although the age ranges for the 2 cohorts were the same (53-74 years), the proportion of those in ARIC aged 65 years and older $(43.5 \%)$ was significantly higher than in the hemophilia cohort (25\%). There were other significant differences between the 2 cohorts, as shown in Table 4. The patients with hemophilia were more likely to be of white race, have hypertension, have a family history of CVD, and have an elevated hsCRP and creatinine. Participants in the ARIC cohort were more likely to have ever smoked, have an elevated waist circumference, and be receiving antihypertensive medications. In a logistic regression model, the odds of having CVD in men with hemophilia vs ARIC, after adjusting for the effects of age, race, ever smoking, family history of CVD, hypertension, diabetes mellitus, dyslipidemia, elevated hsCRP, and

Table 3. Model-predicted odds ratios for potential CVD risk factors among patients with hemophilia

\begin{tabular}{|c|c|c|c|}
\hline Effect & Odds ratio & $\mathbf{9 5} \%$ confidence interval & $\boldsymbol{P}$ \\
\hline Age $\geq 65$ y vs age $<65 y$ & 1.18 & $0.40-3.48$ & .761 \\
\hline White vs non-white & 0.19 & $0.05-0.75$ & .018 \\
\hline Ever smoker vs no smoker & 3.12 & $1.20-8.11$ & .020 \\
\hline Positive family history of CVD vs no family history & 1.89 & $0.76-4.73$ & .172 \\
\hline HTN vs no HTN & 5.07 & $1.27-20.21$ & .022 \\
\hline Dyslipidemia vs no dyslipidemia & 3.87 & 1.07-13.96 & .039 \\
\hline DM vs no DM & 1.20 & $0.42-3.39$ & .734 \\
\hline Hemophilia A vs B & 0.44 & $0.16-1.17$ & .100 \\
\hline HIV vs no HIV & 1.49 & $0.41-5.41$ & .548 \\
\hline Inhibitor vs no inhibitor & 9.18 & $1.26-66.90$ & .029 \\
\hline Severe vs moderate hemophilia with HCV & 0.81 & 0.19-3.37 & .879 \\
\hline Severe vs moderate hemophilia without HCV & 0.23 & $0.06-0.99$ & .049 \\
\hline
\end{tabular}

CVD, cardiovascular disease; DM, diabetes mellitus; HCV, hepatitis C virus; HIV, human immunodeficiency virus; HTN, hypertension. 
Table 4. Comparison between the ARIC, NHANES, and hemophilia cohorts

\begin{tabular}{|c|c|c|c|c|c|}
\hline Variable & Hemophilia, \% & ARIC, \% & ARIC vs hemophilia, $P$ & NHANES, \% & NHANES vs hemophilia, $P$ \\
\hline CVD (ARIC definition)* & 15.0 & 25.8 & $<.001$ & & \\
\hline CVD (NHANES definition)* & 10.0 & & & 17.9 & .003 \\
\hline ASA use in patients with CVD & 10.0 & 59.0 & $<.001$ & 22.7 & .024 \\
\hline Age $\geq 65$ y & 25.0 & 43.5 & $<.001$ & 37.2 & $<.001$ \\
\hline White & 88.5 & 82.4 & .013 & & \\
\hline Hypertension & 64.5 & 46.3 & $<.001$ & 55.0 & .007 \\
\hline Antihypertensive medication (in patients with hypertension) & 55.8 & 76.4 & $<.001$ & 85.0 & $<.001$ \\
\hline Dyslipidemia & 63.5 & 63.7 & .477 & $56.8+$ & .042 \\
\hline Use of lipid-lower medication (in patients with dyslipidemia) & 27.6 & 25.6 & .307 & $67.3+$ & $<.001$ \\
\hline Diabetes mellitus & 21.0 & 18.4 & .177 & 28.0 & .022 \\
\hline Diabetes medication (in patients with diabetes) & 45.2 & 55.7 & .091 & 75.9 & $<.001$ \\
\hline Ever smoker & 48.5 & 72.7 & $<.001$ & 61.6 & $<.001$ \\
\hline Family history of CVD & 43.5 & 22.0 & $<.001$ & & \\
\hline Obesity & 29.5 & 31.5 & .275 & 34.7 & .081 \\
\hline Increased waist circumference & 30.0 & 48.8 & $<.001$ & 50.5 & $<.001$ \\
\hline Elevated creatinine & 26.8 & 7.4 & $<.001$ & & \\
\hline Elevated CRP & 9.8 & 5.0 & .002 & & \\
\hline
\end{tabular}

${ }^{*}$ Calculated value by NHANES based on triglyceride and LDL values in $45.2 \%$ and $44.4 \%$ of subjects, respectively.

tSee Methods for definitions.

elevated creatinine, was 0.42 (95\% confidence interval, 0.28 0.63). In other words, if the values of other predictors were equal, men without hemophilia had 2.4 times higher odds of developing CVD.

To compare CVD rates in patients with hemophilia with a more contemporaneous population cohort, we used the NHANES 2013 to 2014 examination (https://wwwn.cdc.gov/nchs/nhanes). About $8.2 \%(n=71)$ of selected NHANES subjects had angina either through self-reporting or Rose questionnaire, $8.6 \%(n=74)$ reported myocardial infarction, and $4.9 \%$ reported a stroke ( $\mathrm{n}=$ 42). Without accounting for $\mathrm{Ml}$ detected via ECG only, or the occurrence of TIA, $17.9 \%(n=154)$ of NHANES patients had CVD, which was significantly higher than for the patients with hemophilia $(P<.001)$, of whom only $10 \%(n=20)$ reported angina $(n=16)$, $\mathrm{Ml}(\mathrm{n}=6)$, or stroke $(\mathrm{n}=1)$.

There were differences in CVD risk factors between the NHANES (ages 53-74 years) and hemophilia subjects (Table 4). Compared with the patients with hemophilia, NHANES subjects were older, had increased waist circumference, and had a history of ever smoking, diabetes, and elevated total cholesterol. Patients with hemophilia were more likely than NHANES subjects to have a low HDL and to have hypertension. However, NHANES estimated triglyceride and LDL values on the basis of data from fewer than $50 \%$ of subjects, and thus may not be a true reflection of the population. Because of different measures used between the cohorts, we could not apply the logistic regression model used for the ARIC comparison.

\section{Discussion}

In this study, we analyzed the prevalence of cardiovascular disease in older men with moderate and severe hemophilia.
Although CVD risk factors were common in these men with hemophilia, cardiac events were less common than in similarly aged US male populations. This may not be explained by differences in atherosclerosis. Studies in men with hemophilia have shown similar atherosclerosis, as measured by carotid and femoral artery intimamedia thickness and on autopsy, although these studies included many men with milder hemophilia or untreated HIV infections and may not reflect findings in our population. ${ }^{13-15,21,22}$ Given the role of FVIII and FIX in thrombin generation, there may be less thrombus formation with an acute event, such as plaque rupture, decreasing the likelihood of an acute event.

The demographics of our subjects were generally representative of the hemophilia population in the United States. In contrast to younger patients with hemophilia, there were proportionally more patients with hemophilia B. Our population characteristics are reflected in the American Thrombosis and Hemostasis Network data set, in which men aged 50 to 74 years represent $15.5 \%$ of those with moderate or severe hemophilia $B$, but only $9.2 \%$ with moderate or severe hemophilia $A{ }^{5}$ This is likely a result of the higher rate of HIV infection and subsequent death in those being treated with contaminated FVIII-containing vs FIX-containing concentrates in the past. ${ }^{23}$ This could affect our analysis, although no difference in risk factors or CVD events by type of hemophilia was noted. The reason why there were proportionally more African American subjects with severe disease is not clear, but may have been influenced by the small number of African American subjects enrolled in the study.

We did not find a difference in the prevalence of CVD by treatment approach (prophylaxis vs on-demand therapy). One could hypothesize that patients using routine prophylactic factor infusions would have less protection from CVD, given higher overall factor activity 
levels. Alternatively, prophylactic therapy could prevent joint bleeding and associated inflammation, which might increase CVD risk. Although we do not have detailed information on the length of prophylactic therapy, given the ages of the men in our cohort and clinical practice patterns in the United States, the introduction of prophylaxis would likely have been as adults. Thus, our data cannot inform questions around CVD in patients receiving prophylaxis from early childhood.

The patient population we studied is of an age severely affected by factor replacement-related infections with HIV and hepatitis $C$ and $B$ that occurred before adoption of viral-inactivation methodology, beginning in the mid-1980s. ${ }^{24,25}$ Twenty-seven percent of our population was HIV infected. This represents a group that likely infused less at the infection risk time, and thus may have phenotypically less severe disease. In some ways, our cohort represents a "survivor" cohort, and our results could be influenced by factors that contributed to their ability to live long enough to participate in this study. Although HIV and medications for HIV are associated with accelerated CVD in other populations, ${ }^{26}$ we did not find a higher prevalence of CVD in our HIV-positive subjects. Our findings may provide insight into the pathogenesis of HIV and HIV medication-associated CVD, and particularly the role of coagulation in reported events.

Chronic HCV infection has also been associated with an increased risk for CVD, particularly in those with other CVD risk factors. ${ }^{27}$ This was not observed in our population. However, we did find an increased incidence of CVD in patients with moderate vs severe disease in patients who were not $\mathrm{HCV}$ infected. It is possible that a risk associated with $\mathrm{HCV}$, such as chronic inflammation, masks a protective effect of a lower factor level.

As has been described in several other cohort studies, ${ }^{16,28-33}$ we also found an increased prevalence of hypertension compared with ARIC and NHANES subjects in our population. The reason why men with hemophilia have more hypertension, as well as higher blood pressures, compared with the general population is unknown, but may be linked to vascular abnormalities in arthropathic joints. ${ }^{34} \mathrm{We}$ found a higher prevalence of abnormal serum creatinine in our cohort compared with ARIC subjects; this was not collected for the NHANES cohort. Given the link between hypertension and renal disease, ${ }^{35}$ it is possible that renal disease is an etiology. von Drygalski et al also found an association between hypertension and elevated creatinine in subjects with hemophilia, which was similar to that found in NHANES subjects. ${ }^{16}$ The large Advance study of men with hemophilia (all severities) in Europe also found an association between hypertension and renal dysfunction. ${ }^{36}$ However, such an association has not been found in all cohort studies. ${ }^{30} \mathrm{~A}$ follow-up study to our current study is underway to better define renal disease in hemophilia. One consideration may be the role of hematuria. Patients with hemophilia frequently have hematuria, which is treated conservatively unless there is significant blood loss. ${ }^{37}$ However, an association with hematuria and hypertension has not been found in 2 studies, ${ }^{32,36}$ so another etiology may be present. If an association between decreased renal function and hypertension is confirmed, further measures to control blood pressure and prevent renal disease would be warranted. If hematuria appears to increase the risk for subsequent renal disease, population characteristics should be explored further to explain differences in findings between studies.

Our patients reported a low usage of antihypertensive therapy. The Centers for Disease Control and Prevention has reported that approximately $50 \%$ of people with hypertension achieve blood pressure control with medication. ${ }^{38}$ In our study, only $36 \%$ of patients with hypertension were receiving antihypertensive medication. Hypertension is reported in younger men with hemophilia as well, with poor control. ${ }^{16}$ It is important that hemophilia treaters inform patients and their primary care providers of the increased prevalence of hypertension in hemophilia, screen for hypertension at hemophilia clinic visits, and facilitate appropriate care.

In our cohort, 30 subjects met the definition of CVD, but only 4 were receiving low-dose aspirin, 3 as secondary and 1 started before, as primary prevention. This was lower than those in the ARIC and NHANES cohorts. Data from the National Health Interview Survey in 2012 found that $70 \%$ of people with CVD were receiving low-dose aspirin. ${ }^{39}$ Patients with hemophilia of this era were strongly advised not to take aspirin when they were younger, and for this reason, many have aspirin in their medical record as an "allergy." This advice was instituted when aspirin was used at much higher doses, and before the cardiovascular benefits of low-dose aspirin had been described. There are minimal data on the safety of low-dose aspirin in patients with hemophilia, although expert opinion has recommended that patients receiving low-dose aspirin with FVIII below 1\% use prophylaxis, but not those with higher factor levels. ${ }^{40-42}$ We have no data to suggest that low-dose aspirin has less cardioprotective effect in men with hemophilia than in the general population. Given that $30 \%$ of our subjects were receiving regular prophylactic therapy, and approximately half of subjects had moderate disease, it is likely that many could tolerate low-dose aspirin without a change in treatment regimen. In addition, given that we found no difference in CVD events between those receiving prophylaxis vs on-demand therapy, underlying atherosclerotic vascular disease should not be a reason to withhold prophylaxis, and prophylaxis, or other regimens such as gene therapy, would allow use of more optimal cardiovascular medications and interventions.

Six of the 9 patients with neutralizing antibodies (inhibitors) to FVIII had CVD in our hemophilia cohort. The presence of an inhibitor was significantly associated with CVD in our analysis (odds ratio, 9.18; $95 \%$ confidence interval, 1.26-66.90; $P=.029$ ). This finding was likely influenced by the small number of inhibitor patients, which is reflected in the wide confidence interval, and this makes it unclear whether it is a true physiological finding. The patients with inhibitors treated bleeding episodes with bypassing agents, which contain activated clotting factors. However, no acute thrombotic event temporally associated with bypassing agent treatment was recorded in the study.

The strengths of our study are that it was multicenter and focused on men with more severe disease. If a low baseline factor level is protective against CVD, it would be more likely to be detected in a population with more severe disease, as the protective effect could be diluted or masked in cohorts that include patients with mildly decreased factor levels. This is particularly true of rare diseases such as hemophilia, for which subject numbers in studies tend to be low. Our study also used medical records to confirm CVD diagnoses, central laboratory testing for lipids, and hsCRP and central reading of ECGs.

A major limitation of our study was the absence of an active control group. We chose the ARIC cohort for comparison and used the same definition of CVD because of characteristics similar to those in our older hemophilia population and study measurements that we 
could obtain, although the cohort is noncontemporaneous. In the NHANES study of 21472 adults aged 40 years and older, although the prevalence of coronary heart disease decreased during that time, the reduction was seen mainly in individuals without established CVD risk factors. ${ }^{43}$ However, there were differences in risk factors between cohorts, and prevalence of specific diseases has changed over time. The NHANES cohort is more contemporaneous, but fewer data are available to compare with the hemophilia cohort, and risk factors also differ between the populations.

Another limitation is potential bias in subject selection. Each HTC was to approach all patients who met inclusion criteria at their HTC. However, patients with more medical problems may visit the HTC more frequently and be available for study. In addition, our population was limited to those seen in HTCs, which is estimated to represent $70 \%$ to $80 \%$ of the overall hemophilia population in the United States. Both these points could potentially bias our cohort to those with more comorbidities that might result in overestimation of CVD in the population.

As noted earlier, a prospective study to further investigate the significantly increased rate of renal dysfunction noted in our hemophilia population, and to follow this cohort as they continue to age, is underway. In addition, more data are needed to determine the role of inhibitors as a potential risk factor for CVD. Given the prevalence of CVD risk factors in men with hemophilia and low rates of hypertension control and aspirin use, efforts to optimize care are needed. Patients with hemophilia who present with acute coronary syndromes often receive nonstandard care, which may negatively affect outcomes. ${ }^{41,44}$

In summary, although patients with moderate and severe hemophilia appear to have some protection from cardiovascular thrombotic events compared with the general population, cardiovascular risk factors are common, including an increased prevalence of hypertension. Thus, on an individual-patient level, optimal regimens for cardioprevention are warranted. For example, only $28 \%$ of our patients with dyslipidemia were receiving statins, medications that are not associated with increased bleeding. A meta-analysis of 27 randomized trials of pravastatin therapy in patients at low risk for vascular disease found that for every $40 \mathrm{mg} / \mathrm{dL}$ decrease in LDL cholesterol, there was a $20 \%$ to $25 \%$ decrease in major cardiovascular events. Thus, given the occurrence of CVD in men with hemophilia, and the challenges in its treatment in this population, measures directed at screening for and managing cardiovascular risk factors and optimizing management of CVD in this population are needed.

\section{Acknowledgments}

The authors thank the research coordinators at each site: Natasia Weiss and Jacqueline Buckley (Indiana Hemophilia and Thrombosis Center), Helena Jacobs (Georgetown University), Rebecca Hauke (University of Michigan), Gail Long (Hemophilia Center of Central Pennsylvania/Penn State Milton S. Hershey Medical Center), Stephanie Whitten (Emory University Comprehensive Hemophilia Program), Manny Mangilit (Hemophilia Treatment Center, Orthopaedic Hospital), Mary Kelty (Penn Comprehensive Hemophilia and Thrombosis Program/Hospital of the University of Pennsylvania), Jacqueline Washington and Judith Kadosh (Hemophilia Center of Western Pennsylvania), Deborah Bennett (Mary M. Gooley Hemophilia Center, Inc./Rochester General Hospital), Carol deKernion (Louisiana Comprehensive Hemophilia Care Center/Tulane University Health Science Center), Robin Kellerman (Hemophilia and Thrombosis Center/University of North Carolina), Karen Stephany (Blood Center of Wisconsin), Molly Brown and Kristi Norton (Hemophilia and Thrombosis Center/University of Colorado), Madeline Cantini and Krishna Cannon (Gulf States Hemophilia and Thrombophilia Center), Ilana Levin (Hemophilia Treatment Center, University of California, San Diego), Mary Jones (The Vanderbilt Hemostasis/ Hemophilia Clinic), Melanie Heinlein (The Ohio State University Medical Center Hemophilia Treatment Center), and Linda Mueller and Nancy Sullivan (Henry Ford Hemophilia and Thrombosis Treatment Center)

The authors acknowledge research funding from the Centers for Disease Control (B.A.K. and the American Thrombosis and Hemostasis Network) and from the US Department of Health and Human Services (H30MC24049) for use of Washington Center for Bleeding Disorders $340 B$ program income for research (B.A.K.).

\section{Authorship}

Contribution: S.L.S. and B.A.K. devised the study and received funding; D.C. and C.W. further modified the study and data collection; D.C. provided statistical analysis; M.R., C.M.K., D.Q., A.D.S., N.S.K., M.J.M.-J., A.C., C.K., T.-F.W., M.E.E., P.K., A.v.D., J.C.G., A.W., P.K., M.A.E., C.L., and S.G. gave input into study design, collected data, and gave input into the manuscript; and M.C. analyzed ECGs and gave input into the manuscript.

Conflict-of-interest disclosure: The authors declare no competing financial interests.

Correspondence: Barbara A. Konkle, Bloodworks Northwest, 921 Terry Ave, Seattle WA 98104; e-mail: barbarak@bloodworksnw. org.

\section{References}

1. Mannucci PM, Tuddenham EGD. The hemophilias-from royal genes to gene therapy. N Eng/ J Med. 2001;344(23):1773-1779.

2. Josephson N. The hemophilias and their clinical management. Hematology Am Soc Hematol Educ Program. 2013;2013:261-267.

3. Darby SC, Kan SW, Spooner RJ, et al. Mortality rates, life expectancy, and causes of death in people with hemophilia A or B in the United Kingdom who were not infected with HIV. Blood. 2007;110(3):815-825.

4. Manco-Johnson MJ, Soucie JM, Gill JC; Joint Outcomes Committee of the Universal Data Collection, US Hemophilia Treatment Center Network. Prophylaxis usage, bleeding rates, and joint outcomes of hemophilia, 1999 to 2010: a surveillance project. Blood. 2017;129(17):2368-2374.

5. American Thrombosis and Hemostasis Research Report. https://athn.org/what-we-do/national-projects/athndataset.html. Accessed 11 January 2018.

6. Plug I, Van Der Bom JG, Peters M, et al. Mortality and causes of death in patients with hemophilia, 1992-2001: a prospective cohort study. J Thromb Haemost. 2006;4(3):510-516. 
7. Kulkarni R, Soucie JM, Evatt BL; Hemophilia Surveillance System Project Investigators. Prevalence and risk factors for heart disease among males with hemophilia. Am J Hematol. 2005;79(1):36-42.

8. Biere-Rafi S, Zwiers M, Peters M, et al. The effect of haemophilia and von Willebrand disease on arterial thrombosis: a systematic review. Neth J Med. 2010;68(5):207-214.

9. Ragni MV, Moore CG. Atherosclerotic heart disease: prevalence and risk factors in hospitalized men with haemophilia A. Haemophilia. 2011;17(6): 867-871.

10. Lövdahl S, Henriksson KM, Baghaei F, et al. Incidence, mortality rates and causes of deaths in haemophilia patients in Sweden. Haemophilia. 2013;19(3): 362-369.

11. Fransen van de Putte DE, Fischer K, Makris M, et al. History of non-fatal cardiovascular disease in a cohort of Dutch and British patients with haemophilia. Eur J Haematol. 2012;89(4):336-339.

12. Pocoski J, Ma A, Kessler CM, Boklage S, Humphries TJ. Cardiovascular comorbidities are increased in U.S. patients with haemophilia A: a retrospective database analysis. Haemophilia. 2014;20(4):472-478.

13. Foley CJ, Nichols L, Jeong K, Moore CG, Ragni MV. Coronary atherosclerosis and cardiovascular mortality in hemophilia. J Thromb Haemost. 2010;8(1): 208-211.

14. Tuinenburg A, Rutten A, Kavousi M, et al. Coronary artery calcification in hemophilia A: no evidence for a protective effect of factor VIII deficiency on atherosclerosis. Arterioscler Thromb Vasc Biol. 2012;32(3):799-804.

15. Bilora F, Zanon E, Petrobelli F, et al. Does hemophilia protect against atherosclerosis? A case-control study. Clin App/ Thromb Hemost. 2006;12(2): 193-198.

16. von Drygalski A, Kolaitis NA, Bettencourt R, et al. Prevalence and risk factors for hypertension in hemophilia. Hypertension. 2013;62(1):209-215.

17. Barnes RFW, Cramer TJ, Sait AS, Kruse-Jarres R, Quon DVK, von Drygalski. The hypertension of hemophilia is not explained by the usual cardiovascular risk factors: results of a cohort study. Int J Hypertens. 2016;2016:2014201.

18. Rose GA. The diagnosis of ischaemic heart pain and intermittent claudication in field surveys. Bull World Health Organ. 1962;27(6):645-658.

19. The ARIC Investigators. The Atherosclerosis Risk in Communities (ARIC) Study: design and objectives. The ARIC investigators. Am J Epidemiol. 1989; 129(4):687-702.

20. Zhang ZM, Rautaharju PM, Prineas RJ, et al. Race and sex differences in the incidence and prognostic significance of silent myocardial infarction in the atherosclerosis risk in communities (ARIC) study. Circulation. 2016;133(22):2141-2148.

21. Zwiers M, Lefrandt JD, Mulder DJ, et al. Coronary artery calcification score and carotid intima-media thickness in patients with hemophilia. $J$ Thromb Haemost. 2012;10(1):23-29.

22. Biere-Rafi S, Tuinenburg A, Haak BW, et al. Factor VIII deficiency does not protect against atherosclerosis. J Thromb Haemost. 2012;10(1):30-37.

23. Goedert JJ, Kessler CM, Aledort LM, et al. A prospective study of human immunodeficiency virus type 1 infection and the development of AIDS in subjects with hemophilia. N Engl J Med. 1989;321(17):1141-1148.

24. Mazepa MA, Monahan PE, Baker JR, Riske BK, Soucie JM; US Hemophilia Treatment Center Network. Men with severe hemophilia in the United States: birth cohort analysis of a large national database. Blood. 2016;127(24):3073-3081.

25. Siboni SM, Mannucci PM, Gringeri A, et al; Italian Association of Haemophilia Centres (AICE). Health status and quality of life of elderly persons with severe hemophilia born before the advent of modern replacement therapy. J Thromb Haemost. 2009;7(5):780-786

26. Hemkens LG, Bucher HC. HIV infection and cardiovascular disease. Eur Heart J. 2014;35(21):1373-1381.

27. Petta $S$, Maida M, Macaluso FS, et al. Hepatitis $C$ virus infection is associated with increased cardiovascular mortality: a meta-analysis of observational studies. Gastroenterology. 2016;150(1):145-155.e4, quiz e15-e16.

28. Rosendaal FR, Briët E, Stibbe J, et al. Haemophilia protects against ischaemic heart disease: a study of risk factors. Br J Haematol. 1990;75(4):525-530.

29. Biere-Rafi S, Baarslag MA, Peters M, et al. Cardiovascular risk assessment in haemophilia patients. Thromb Haemost. 2011;105(2):274-278.

30. Fransen van de Putte DE, Fischer K, Makris M, et al. Increased prevalence of hypertension in haemophilia patients. Thromb Haemost. 2012;108(4): 750-755.

31. Sait AS, Kuo A, Bettencourt R, Bergstrom J, Allison M, von Drygalski A. Risk assessment for coronary heart disease in patients with haemophilia: a single centre study in the United States. Haemophilia. 2014;20(6):763-770.

32. Sun HL, Yang M, Sait AS, von Drygalski A, Jackson S. Haematuria is not a risk factor of hypertension or renal impairment in patients with haemophilia. Haemophilia. 2016;22(4):549-555.

33. Fransen van de Putte DE, Fischer K, Makris M, et al. Unfavourable cardiovascular disease risk profiles in a cohort of Dutch and British haemophilia patients. Thromb Haemost. 2013;109(1):16-23.

34. Barnes RFW, Cramer TJ, Hughes TH, von Drygalski A. The hypertension of hemophilia is associated with vascular remodeling in the joint. Microcirculation. 2017;24(7):e12387.

35. Weir MR. Hypertension and the kidney: perspectives on the relationship of kidney disease and cardiovascular disease. Clin J Am Soc Nephrol. 2009; 4(12):2045-2050.

36. Holme PA, Combescure C, Tait RC, Berntorp E, Rauchensteiner S, de Moerloose P; ADVANCE Working Group. Hypertension, haematuria and renal functioning in haemophilia - a cross-sectional study in Europe. Haemophilia. 2016;22(2):248-255.

37. Quon DV, Konkle BA. How we treat: haematuria in adults with haemophilia. Haemophilia. 2010;16(4):683-685. 
38. Farley TA, Dalal MA, Mostashari F, Frieden TR. Deaths preventable in the U.S. by improvements in use of clinical preventive services. Am J Prev Med. 2010;38(6):600-609.

39. Fang J, George MG, Gindi RM, et al. Use of low-dose aspirin as secondary prevention of atherosclerotic cardiovascular disease in US adults (from the National Health Interview Survey, 2012). Am J Cardiol. 2015;115(7):895-900.

40. Mannucci PM, Schutgens REG, Santagostino E, Mauser-Bunschoten EP. How I treat age-related morbidities in elderly persons with hemophilia. Blood. 2009;114(26):5256-5263.

41. Staritz P, de Moerloose P, Schutgens R, Dolan G; ADVANCE Working Group. Applicability of the European Society of Cardiology guidelines on management of acute coronary syndromes to people with haemophilia - an assessment by the ADVANCE Working Group. Haemophilia. 2013;19(6): 833-840.

42. Angelini D, Konkle BA, Sood SL. Aging among persons with hemophilia: contemporary concerns. Semin Hematol. 2016;53(1):35-39.

43. Yoon SS, Dillon CF, Illoh K, Carroll M. Trends in the prevalence of coronary heart disease in the U.S.: national health and nutrition examination survey, 2001-2012. Am J Prev Med. 2016;51(4):437-445.

44. Fogarty PF, Mancuso ME, Kasthuri R, et al; Global Emerging Hemophilia Panel (GEHEP). Presentation and management of acute coronary syndromes among adult persons with haemophilia: results of an international, retrospective, 10-year survey. Haemophilia. 2015;21(5):589-597. 